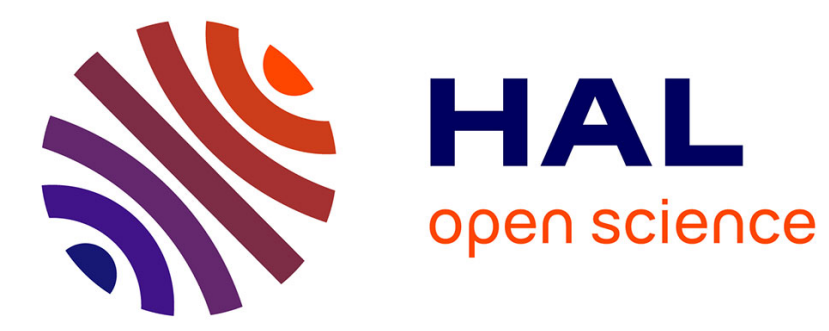

\title{
Learning-based Compositional Verification for Synchronous Probabilistic Systems
}

\author{
Lu Feng, Tingting Han, Marta Kwiatkowska, David Parker
}

\section{To cite this version:}

Lu Feng, Tingting Han, Marta Kwiatkowska, David Parker. Learning-based Compositional Verification for Synchronous Probabilistic Systems. 9th International Symposium on Automated Technology for Verification and Analysis (ATVA'11), 2011, Taipei, Taiwan. pp.511-521. hal-00647061

\section{HAL Id: hal-00647061 \\ https://hal.inria.fr/hal-00647061}

Submitted on 30 Jan 2012

HAL is a multi-disciplinary open access archive for the deposit and dissemination of scientific research documents, whether they are published or not. The documents may come from teaching and research institutions in France or abroad, or from public or private research centers.
L'archive ouverte pluridisciplinaire HAL, est destinée au dépôt et à la diffusion de documents scientifiques de niveau recherche, publiés ou non, émanant des établissements d'enseignement et de recherche français ou étrangers, des laboratoires publics ou privés. 


\title{
Learning-based Compositional Verification for Synchronous Probabilistic Systems
}

\author{
Lu Feng, Tingting Han, Marta Kwiatkowska, and David Parker \\ Department of Computer Science, University of Oxford, Oxford, OX1 3QD, UK
}

\begin{abstract}
We present novel techniques for automated compositional verification of synchronous probabilistic systems. First, we give an assume-guarantee framework for verifying probabilistic safety properties of systems modelled as discretetime Markov chains. Assumptions about system components are represented as probabilistic finite automata (PFAs) and the relationship between components and assumptions is captured by weak language inclusion. In order to implement this framework, we develop a semi-algorithm to check language inclusion for PFAs and a new active learning method for PFAs. The latter is then used to automatically generate assumptions for compositional verification.
\end{abstract}

\section{Introduction}

Probabilistic model checking is a formal verification technique for analysing quantitative properties of systems that exhibit stochastic behaviour. A key challenge in this area is scalability, motivating the development of compositional verification methods that decompose the analysis of a large system model into smaller sub-tasks. We focus on the assume-guarantee paradigm, in which each system component is analysed under an assumption about the other component(s) it is composed with. After checking that the assumption is satisfied, proof rules are used to deduce properties of the overall system.

Several assume-guarantee frameworks for verifying probabilistic systems have been proposed, mainly for models with both probabilistic and nondeterministic behaviour [1[13 10]. The main difficulty when developing such a framework is formulating an appropriate notion of assumptions that can support compositional reasoning. Our goal is to develop assume-guarantee techniques for probabilistic model checking that are practical, efficient and fully-automated. This means that assumptions should ideally: (i) be expressive enough for practical applications; (ii) allow efficient, fully-automated verification; and (iii) be amenable to automatic generation.

One promising direction is the framework of [13] (and its extensions in [10]9]). In [13], assumptions are probabilistic safety properties (e.g. "event A always occurs before event B with probability at least 0.98 ") and [10] generalises this to boolean combinations of $\omega$-regular and reward properties. In both cases, this yields efficiently checkable assumptions and the approaches were successfully implemented and applied to some large case studies. Furthermore, [9] shows how to automatically generate probabilistic safety property assumptions [13] using learning techniques based on L*.

In this work, we continue to develop probabilistic assume-guarantee techniques in which assumptions can be automatically generated via learning. In particular, our focus is on using a more expressive class of assumptions. Probabilistic safety property 
assumptions [13] can only capture a limited amount of information about a component, restricting the cases where assume-guarantee reasoning can be applied. The framework of [13] is incomplete in the sense that, if the property being verified is true, there does not necessarily exists an assumption that can be used to verify it compositionally.

This paper proposes novel techniques for compositional probabilistic verification in which assumptions are probabilistic finite automata (PFAs) [15]. Unlike [13]10], our approach is complete. Furthermore, as in [10], we use learning to automatically generate assumptions. PFAs represent weighted languages, mapping finite words to probabilities. In our framework, an assumption about a system component $M$ is represented by a PFA that gives upper bounds on the probabilities of traces being observed in $M$. This is an inherently linear-time relation, which is well-known to be difficult to adapt to compositional techniques for systems that exhibit both probabilistic and nondeterministic behaviour [16]. So, in the present work, we restrict our attention to fully probabilistic systems. To do so, we model components as probabilistic I/O systems (PIOSs), which, when combined through synchronous parallel composition, result in a (fully probabilistic) discrete-time Markov chain (DTMC). The relation between a PIOS $M$ and a PFA $A$ representing an assumption about $M$ is captured by weak language inclusion. Based on this, we give an asymmetric proof rule for verifying probabilistic safety properties on a DTMC composed of two PIOSs.

In order to implement our framework, we give an algorithm to check weak language inclusion, reducing it to the existing notion of (strong) language inclusion for PFAs. Although checking PFA language equivalence (that each word maps to the same probability) is decidable in polynomial time [1877], checking language inclusion is undecidable [5]. We propose a semi-algorithm, inspired by [18], to check language inclusion; in the case where the check fails, a minimal counterexample is produced.

We also develop a novel technique for learning PFAs, which we use to automatically generate assumptions for our framework. Our algorithm, like $\mathrm{L}^{*}$, is based on active learning, posing queries in an interactive fashion about the PFA to be generated. Several active PFA learning algorithms exist [12 [17|4] but are not suitable for our needs: [12] applies to a restricted class of PFAs, [17] needs to know the size of the PFA in advance, and [4] actually learns multiplicity automata, which may contain negative values.

Full version: For an extended version of this paper, including additional details, explanations and running examples, experimental results and proofs, see [8].

\section{Preliminaries}

We first briefly describe probabilistic finite automata and discrete-time Markov chains. We use $S$ Dist $(S)$ to denote the set of probability sub-distributions over set $S, \eta_{s}$ for the point distribution on $s \in S$, and $\mu_{1} \times \mu_{2}$ for the product distribution of $\mu_{1}$ and $\mu_{2}$.

Definition 1 (PFA). A probabilistic finite automaton $(P F A)$ is a tuple $A=(S, \bar{s}, \alpha, \mathbf{P})$, where $S$ is a finite set of states, $\bar{s} \in S$ is an initial state, $\alpha$ is an alphabet and $\mathbf{P}: \alpha \rightarrow$ $(S \times S \rightarrow[0,1])$ is a function mapping actions to transition probability matrices. For each $a \in \alpha$ and $s \in S, \sum_{s^{\prime} \in S} \mathbf{P}(a)\left[s, s^{\prime}\right] \in[0,1]$.

A PFA $A$ defines a mapping $\operatorname{Pr}^{A}: \alpha^{*} \rightarrow[0,1]$ giving the probability of accepting each finite word $w \in \alpha^{*}$. Intuitively, the probability $\operatorname{Pr}^{A}(w)$ for a word $w=a_{1} \cdots a_{n}$ is 
determined by tracing paths through $A$ that correspond to $w$, with $\mathbf{P}(a)\left[s, s^{\prime}\right]$ giving the probability to move from $s$ to $s^{\prime}$ on reading $a$. More precisely, we let $\iota$ be an $S$-indexed 0 -1 row vector with $\iota[s]=1$ if and only if $s=\bar{s}, \boldsymbol{\kappa}$ be an $S$-indexed column vector of 1s and $\mathbf{P}(w)=\mathbf{P}\left(a_{1}\right) \cdots \mathbf{P}\left(a_{n}\right)$. Then, we define $\operatorname{Pr}^{A}(w)=\iota \mathbf{P}(w) \boldsymbol{\kappa}$.

Definition 2 (Language inclusion/equivalence). Given two PFAs $A_{1}$ and $A_{2}$ with the same alphabet $\alpha$, we say $A_{1}$ and $A_{2}$ are related by (strong) language inclusion (resp. language equivalence), denoted $A_{1} \sqsubseteq A_{2}$ (resp. $A_{1} \equiv A_{2}$ ), if for every word $w \in \alpha^{*}$, $\operatorname{Pr}^{A_{1}}(w) \leqslant \operatorname{Pr}^{A_{2}}(w)\left(\right.$ resp. $\left.\operatorname{Pr}^{A_{1}}(w)=\operatorname{Pr}^{A_{2}}(w)\right)$.

Definition 3 (DTMC). A discrete-time Markov chain (DTMC) is a tuple $D=(S, \bar{s}, \alpha, \delta)$, where $S$ is a finite set of states, $\bar{s} \in S$ is an initial state, $\alpha$ is an alphabet of action labels and $\delta: S \times(\alpha \cup\{\tau\}) \rightarrow S D i s t(S)$ is a (partial) probabilistic transition function, such that, for any $s, \delta(s, a)$ is defined for at most one a $\in \alpha \cup\{\tau\}$.

If $\delta(s, a)=\mu$, the DTMC can make a transition, labelled with action $a$, and move to state $s^{\prime}$ with probability $\mu\left(s^{\prime}\right)$. We denote such transitions by $s \stackrel{a}{\rightarrow} \mu$ (or $\stackrel{a}{\rightarrow} s^{\prime}$ ). The DTMC deadlocks when $\delta(s, a)$ is not defined for any $a$, which we denote by $s \not \rightarrow$. We use action label $\tau$ to denote a "silent' (or "internal") transition. A (finite or infinite) path through $D$ is a sequence of transitions $\theta=s_{0} \stackrel{a_{0}}{\rightarrow} s_{1} \stackrel{a_{7}}{\rightarrow} \ldots$ with $s_{0}=\bar{s}$.

In this paper, we consider probabilistic safety properties $\langle G\rangle_{\geqslant p}$, where $G$ is a regular safety property [3], defining a set of "good" executions, and $p \in[0,1]$ is a probability bound. Model checking $\langle G\rangle_{\geqslant p}$ reduces to solving a linear equation system [3].

\section{Assume-Guarantee for Synchronous Probabilistic Systems}

We now define a compositional verification framework for fully probabilistic systems. Components are modelled by probabilistic I/O systems (PIOSs). These exhibit (input) nondeterminism but, when composed synchronously in parallel, result in a DTMC.

Definition 4 (PIOS). A probabilistic I/O system (PIOS) is a tuple $M=(S, \bar{s}, \alpha, \delta)$, where $S$ and $\bar{s}$ are as for DTMCs, and the alphabet $\alpha$ and transition function $\delta: S \times$ $(\alpha \cup\{\tau\}) \rightarrow \operatorname{SDist}(S)$ satisfy the following two conditions: (i) $\alpha$ is partitioned into three disjoint sets of input, output and hidden actions, which we denote $\alpha^{I}, \alpha^{O}$ and $\alpha^{H}$, respectively; input actions $\alpha^{I}$ are further partitioned into $m$ disjoint bundles $\alpha^{I, i}$ $(1 \leqslant i \leqslant m)$ for some $m$; (ii) the set enab $(s) \subseteq \alpha \cup\{\tau\}$ of enabled actions for each state $s$ (i.e. the actions a for which $\delta(s, a)$ is defined) satisfies either $\mid$ enab(s) $\mid=1$ if $\operatorname{enab}(s) \in \alpha^{O} \cup \alpha^{H} \cup\{\tau\}$ or enab $(s)=\alpha^{I, i}$ for some input action bundle $\alpha^{I, i}$.

From any state $s$ of a PIOS $M$, there is either a single transition with an output, hidden or $\tau$ action, or $k$ transitions, each with one action from a particular bundle $\alpha^{I, i}$ comprising $k$ input actions. Transitions and paths in PIOSs are defined as for DTMCs. The probability of a finite path $\theta=s_{0} \stackrel{a_{0}}{\longrightarrow} s_{1} \cdots \stackrel{a_{n-1}}{\longrightarrow} s_{n}$ in $M$ is given by $\operatorname{Pr}^{M}(\theta)=\prod_{i=0}^{n-1} \delta\left(s_{i}, a_{i}\right)\left(s_{i+1}\right)$. Since PIOSs only have nondeterminism on input actions, the probability for a word $w \in(\alpha \cup\{\tau\})^{*}$ is well defined: letting $w d(\theta)$ denote the word $a_{0} \ldots a_{n-1}$ of actions from path $\theta$, we have $\operatorname{Pr}^{M}(w)=\sum_{w d(\theta)=w} \operatorname{Pr}^{M}(\theta)$. Then, letting st: $(\alpha \cup\{\tau\})^{*} \alpha \rightarrow \alpha^{*}$ be the function that removes all $\tau \mathrm{s}$, we define the probability $\operatorname{Pr}_{\tau}^{M}\left(w^{\prime}\right)$ for a $\tau$-free word $w^{\prime} \in \alpha^{*}$ as $\operatorname{Pr}_{\tau}^{M}(w)=\sum_{w=s t\left(w^{\prime}\right)} \operatorname{Pr}^{M}\left(w^{\prime}\right)$. 

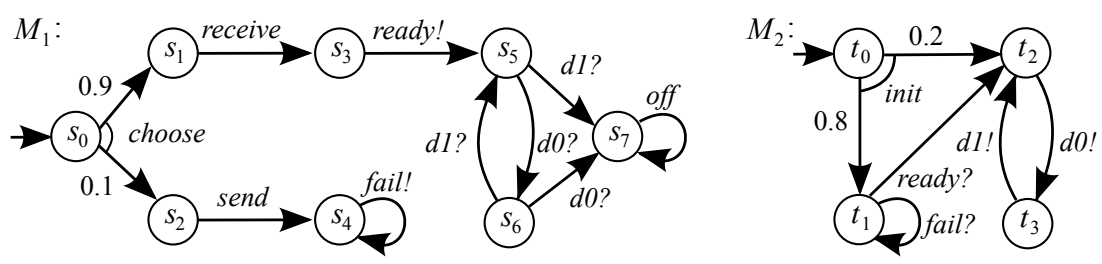

Fig. 1. Running example: two PIOSs $M_{1}$ and $M_{2}$.

Example 1. Fig. 1 depicts two PIOSs $M_{1}$ and $M_{2} . M_{1}$ is a data communicator which chooses (probabilistically) to either send or receive data. This simple example only models receiving; choosing to send results in a failure. $M_{1}$ tells $M_{2}$, a data generator, that it is ready to receive using action ready. $M_{2}$ should then send a sequence of packets, modelled by the alternating actions $d 0$ and $d 1$. If $M_{1}$ has failed, it sends a message fail. $M_{2}$ also has an initialisation step (init), which can fail. With probability 0.8 , it is ready to receive signals from $M_{1}$; otherwise, it just tries to send packets anyway. Input/output actions for $M_{1}, M_{2}$ are labelled with ?/! in the figure; all other actions are hidden. Each PIOS has a single input action bundle: $\alpha_{1}^{I, 1}=\{d 0, d 1\}, \alpha_{2}^{I, 1}=\{$ ready, fail $\}$.

Given PIOSs $M_{1}, M_{2}$ with alphabets $\alpha_{1}, \alpha_{2}$, we say $M_{1}$ and $M_{2}$ are composable if $\alpha_{1}^{I}=\alpha_{2}^{O}, \alpha_{1}^{O}=\alpha_{2}^{I}$ and $\alpha_{1}^{H} \cap \alpha_{2}^{H}=\varnothing$ and define their parallel composition as follows.

Definition 5 (Parallel composition). The parallel composition of composable PIOSs $M_{i}=\left(S_{i}, \bar{s}_{i}, \alpha_{i}, \delta_{i}\right)$ for $i=1,2$ is given by the PIOS $M_{1} \| M_{2}=\left(S_{1} \times S_{2},\left(\bar{s}_{1}, \bar{s}_{2}\right), \alpha, \delta\right)$, where $\alpha=\alpha^{H}=\alpha_{1}^{I} \cup \alpha_{1}^{O} \cup\left(\left(\alpha_{1}^{H} \cup\{\perp\}\right) *\left(\alpha_{2}^{H} \cup\{\perp\}\right)\right)$ and, for $b_{i} \in \alpha_{i}^{H} \cup \tau$ and $a \in \alpha_{1}^{I} \cup \alpha_{1}^{O}$, $\delta$ is defined such that $\left(s_{1}, s_{2}\right) \stackrel{\gamma}{\rightarrow} \mu_{1} \times \mu_{2}$ iff one of the following holds: (i) $s_{1} \stackrel{a}{\rightarrow} \mu_{1}, s_{2} \stackrel{a}{\rightarrow} \mu_{2}, \gamma=a$; (ii) $s_{1} \stackrel{b_{1}}{\rightarrow} \mu_{1}, s_{2} \stackrel{b_{2}}{\rightarrow} \mu_{2}, \gamma=b_{1} * b_{2}$; (iii) $s_{1} \stackrel{b_{1}}{\rightarrow} \mu_{1}, s_{2} \stackrel{a}{\rightarrow}$ (or $s_{2} \not \supset$ ), $\mu_{2}=\eta_{s_{2}}, \gamma=b_{1} * \perp$; (iv) $s_{1} \stackrel{a}{\rightarrow}$ (or $s_{1} \not \supset$ ), $s_{2} \stackrel{b_{2}}{\rightarrow} \mu_{2}, \mu_{1}=\eta_{s_{1}}, \gamma=\perp * b_{2}$.

Notice PIOS $M_{1} \| M_{2}$ has only $\tau$ or hidden actions and can thus be considered a DTMC.

We next introduce our notion of assumptions about PIOSs, for which we use a specific class of PFAs and weak language inclusion, which relaxes the definition of language inclusion for PFAs introduced earlier by ignoring $\tau$ actions.

Definition 6 (Assumption). Let $M$ be a PIOS with alphabet $\alpha=\alpha^{I} \uplus \alpha^{O} \uplus \alpha^{H}$ and input action bundles $\alpha^{I}=\biguplus_{i=1}^{m} \alpha^{I, i}$. An assumption $A$ about $M$ is a PFA $A=$ $(S, \bar{s}, \alpha, \mathbf{P})$ satisfying, for each state $s \in S:$ (i) either all or none of the actions in a bundle $\alpha^{I, i}(1 \leqslant i \leqslant m)$ are enabled in $s$; (ii) $p^{\max }(s) \in[0,1]$, where:

$p^{\max }(s) \stackrel{\text { def }}{=} \sum_{a \in \alpha^{O} \cup \alpha^{H}} \sum_{s^{\prime} \in S} \mathbf{P}(a)\left[s, s^{\prime}\right]+\sum_{i=1}^{m} p_{i}^{\max }(s)$ and $p_{i}^{\max }(s) \stackrel{\text { def }}{=} \max _{a \in \alpha^{I, i}} \sum_{s^{\prime} \in S} \mathbf{P}(a)\left[s, s^{\prime}\right]$

Definition 7 (Weak language inclusion/equivalence). For PIOS $M$ with alphabet $\alpha$ and an assumption $A$ about $M$, we say that $M$ and $A$ are related by weak language inclusion (resp. equivalence), denoted $M \sqsubseteq_{w} A$ (resp. $M \equiv_{w} A$ ), if for every word $w \in \alpha^{*}, \operatorname{Pr}_{\tau}^{M}(w) \leqslant \operatorname{Pr}^{A}(w)\left(\right.$ resp. $\left.\operatorname{Pr}_{\tau}^{M}(w)=\operatorname{Pr}^{A}(w)\right)$.

A valid assumption $A$ for $M$ is one that satisfies $M \sqsubseteq_{w} A$. We can reduce the problem of checking whether this is true to the problem of checking (strong) language inclusion between two PFAs (see Section 4) by the following proposition. 

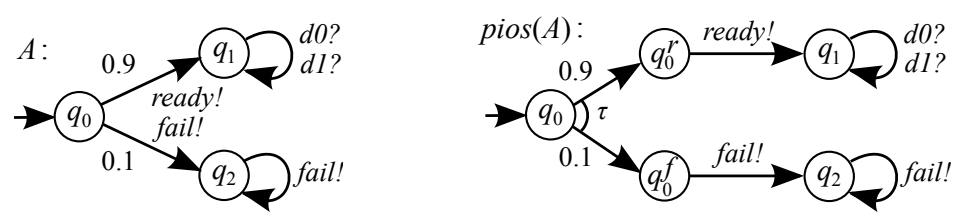

Fig. 2. Assumption $A$ and its PIOS conversion $\operatorname{pios}(A)$.

Proposition 1. Let $M=(S, \bar{s}, \alpha, \delta)$ be a PIOS and $A$ be an assumption about $M$. pfa $(M)=(S, \bar{s}, \alpha \cup\{\tau\}, \mathbf{P})$ is the translation of $M$ to a PFA, where $\mathbf{P}(a)\left[s, s^{\prime}\right]=$ $\delta(s, a)\left(s^{\prime}\right)$ for $a \in \alpha \cup\{\tau\}$. Letting $A^{\tau}$ be the PFA derived from $A$ by adding $\tau$ to its alphabet and a probability $1 \tau$-loop to every state, then: $M \sqsubseteq w A \Leftrightarrow p f a(M) \sqsubseteq A^{\tau}$.

We will also need to perform a conversion in the opposite direction, translating an assumption PFA $A$ into a (weak language) equivalent PIOS, which we denote $\operatorname{pios}(A)$.

Definition 8 (Assumption-to-PIOS conversion). Given assumption $A=(S, \bar{s}, \alpha, \mathbf{P})$, and action partition $\alpha=\left(\biguplus_{i=1}^{m} \alpha^{I, i}\right) \uplus \alpha^{O} \uplus \alpha^{H}$, its conversion to a PIOS is defined as $\operatorname{pios}(A)=\left(S^{\prime}, \bar{s}, \alpha, \delta\right)$, where $S^{\prime}=S \uplus\left\{s^{a} \mid s \in S, a \in \alpha^{H} \cup \alpha^{O}\right\} \uplus\left\{s^{i} \mid s \in\right.$ $S, 1 \leq i \leq m\}$ and $\delta$ is constructed as follows. For any transition $s \stackrel{a}{\longrightarrow} s^{\prime}$, let $p$ denote $\mathbf{P}(a)\left[s, s^{\prime}\right]$ and $p^{\max }(s)$ and $p_{i}^{\max }(s)$ be as defined in Definition 6. Then:

- if $a \in \alpha^{O} \cup \alpha^{H}$, then $\delta(s, \tau)\left(s^{a}\right)=\frac{p}{p^{\max }(s)}$ and $\delta\left(s^{a}, a\right)\left(s^{\prime}\right)=p^{\max }(s)$;

- if $a \in \alpha^{I, i}($ for $1 \leqslant i \leqslant m)$, then $\delta(s, \tau)\left(s^{i}\right)=\frac{p_{i}^{\max }(s)}{p^{\max }(s)}$ and $\delta\left(s^{i}, a\right)\left(s^{\prime}\right)=p \cdot \frac{p^{\max }(s)}{p_{i}^{\max }(s)}$.

Example 2. Consider PIOS $M_{1}$ from Example 1. Fig. 2 shows a valid assumption $A$ for $M_{1}$ (i.e. $M_{1} \sqsubseteq_{w} A$ ) and the corresponding PIOS $\operatorname{pios}(A)$. In $A$, state $q_{0}$ has two output actions leading to respective sub-distributions. Thus $A$ is not a PIOS. In $\operatorname{pios}(A)$, a $\tau$ transition and the states $q_{0}^{\text {ready }}$ and $q_{0}^{\text {fail }}$ (abbreviated to $q_{0}^{r}$ and $q_{0}^{f}$ ) are added.

Now, we describe how to perform compositional verification using our framework. We focus on verifying $\langle G\rangle_{\geqslant p}$ on a DTMC $M_{1} \| M_{2}$ where $M_{i}$ are PIOSs. For simplicity, we will assume that the property refers only to input/output actions of $M_{1}$ and $M_{2}$ and assume that all hidden actions of $M_{1}$ and $M_{2}$ have been renamed as $\tau$ actions, which affects neither the parallel composition $M_{1} \| M_{2}$ nor the probability of satisfying $G$.

An assume-guarantee triple $\langle A\rangle M\langle G\rangle_{\geqslant p}$ means "whenever component $M$ is part of a system satisfying the assumption $A$, the system is guaranteed to satisfy $\langle G\rangle_{\geqslant p}$ ".

Definition 9 (Assume-guarantee triple). If $M$ is a PIOS with alphabet $\alpha, A$ is an assumption about $M$ and $\langle G\rangle_{\geqslant p}$ is a probabilistic safety property, then $\langle A\rangle M\langle G\rangle_{\geqslant p}$ is an assume-guarantee triple, with the following meaning:

$$
\langle A\rangle M\langle G\rangle_{\geqslant p} \Leftrightarrow \forall M^{\prime} .\left(M^{\prime} \sqsubseteq_{w} A \Longrightarrow M^{\prime} \| M=\langle G\rangle_{\geqslant p}\right) .
$$

Using the translation $\operatorname{pios}(A)$ from PFA to PIOS described above, checking whether a triple is true reduces to standard probabilistic model checking (see Section 2).

Proposition 2. For $A, M$ and $\langle G\rangle_{\geqslant p}$ as given in Definition 9 the assume-guarantee triple $\langle A\rangle M\langle G\rangle_{\geqslant p}$ holds if and only if pios $(A) \| M \models\langle G\rangle_{\geqslant p}$. 
Finally, we give an asymmetric assume-guarantee proof rule (in the style of those from [14|13]) for verifying a system $M_{1} \| M_{2}$ compositionally.

Theorem 1. Let $M_{1}, M_{2}$ be PIOSs, A an assumption for $M_{1}$ and $\langle G\rangle_{\geqslant p}$ a probabilistic safety property for $M_{1} \| M_{2}$. Then the following proof rule holds:

$$
\frac{M_{1} \sqsubseteq_{w} A \text { and }\langle A\rangle M_{2}\langle G\rangle_{\geqslant p}}{M_{1} \| M_{2} \models\langle G\rangle_{\geqslant p}} \text { (Asym-PIOS) }
$$

Thus, given an appropriate assumption $A$ about $M_{1}$, we can decompose the verification of $M_{1} \| M_{2}$ into two sub-problems: checking weak language inclusion between $M_{1}$ and $A$; and checking that $\langle A\rangle M_{2}\langle G\rangle_{\geqslant p}$. The former, as shown in Proposition 1 reduces to (strong) language inclusion on PFAs, which we discuss in the next section. The latter, as shown in Proposition 2, requires construction of the DTMC pios $(A) \| M_{2}$ and then application of standard probabilistic model checking techniques.

Example 3. Consider probabilistic safety property $\langle G\rangle_{\geqslant 0.9}$, where $G$ means " fail never occurs". We can check this on running example $M_{1} \| M_{2}$ using assumption $A$ from Example 22 Since $M_{1} \sqsubseteq_{w} A$, we just need to check that $\operatorname{pios}(A) \| M_{2} \models\langle G\rangle_{\geqslant 0.9}$. As $\operatorname{pios}(A) \| M_{2}$ has a single path $\left(q_{0} t_{0}\right) \stackrel{\tau * i n i t, 0.08}{\longrightarrow}\left(q_{2} t_{1}\right) \stackrel{\text { fail, } 1}{\longrightarrow}\left(q_{4} t_{1}\right) \cdots$ containing fail with probability $0.08,\langle G\rangle_{\geqslant 0.9}$ is satisfied (since $1-0.08 \geqslant 0.9$ ) and we are done.

Completeness. Our framework is complete in the sense that, if $M_{1} \| M_{2} \models\langle G\rangle_{\geqslant p}$, we can always find an assumption $A$ to apply Theorem 1 by converting $M_{1}$ to a PFA.

\section{Deciding Language Inclusion for PFAs}

As discussed above, verifying whether a component satisfies an assumption in our framework reduces to checking language inclusion between PFAs, i.e. deciding whether two PFAs $A_{1}$ and $A_{2}$ over the same alphabet $\alpha$ satisfy $A_{1} \sqsubseteq A_{2}$. In this section, we propose a semi-algorithm for performing this check. If $A_{1} \sqsubseteq A_{2}$ does not hold, then the algorithm is guaranteed to terminate and return a lexicographically minimal word as a counterexample; but if $A_{1} \sqsubseteq A_{2}$ does hold, then the algorithm may not terminate. The latter case is unavoidable since the problem is undecidable (see [8]).

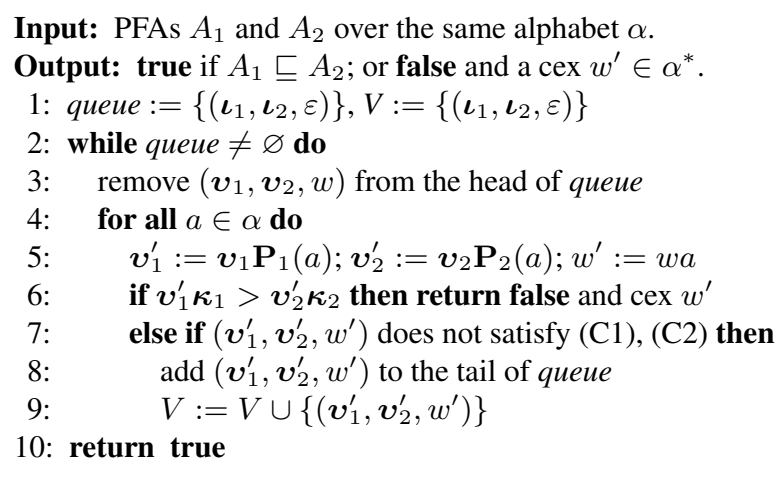

Fig. 3. Semi-algorithm for deciding PFA language inclusion
Fig. 3 shows the semialgorithm to decide if $A_{1} \sqsubseteq$ $A_{2}$, where $A_{i}=\left(S_{i}, \bar{s}_{i}, \alpha, \mathbf{P}_{i}\right)$ for $i=1,2$. We also define $\boldsymbol{\iota}_{i}$ and $\boldsymbol{\kappa}_{i}$ as in Section 2 . Inspired by the language equivalence decision algorithm in [18], our method proceeds by expanding a tree. Each node of the tree is of the form $\left(\boldsymbol{v}_{1}, \boldsymbol{v}_{2}, w\right)$, where $w$ is a word and $\boldsymbol{v}_{i}=$ $\boldsymbol{\iota}_{i} \mathbf{P}_{i}(w)$ (for $i=1,2$ ) is the vector of probabilities of 
reaching each state via word $w$ in $A_{i}$. Note that $\boldsymbol{v}_{i} \boldsymbol{\kappa}_{i}$ is the probability of PFA $A_{i}$ accepting the word $w$. The root of the tree is $\left(\iota_{1}, \iota_{2}, \varepsilon\right)$, where $\varepsilon$ is the empty word. As shown in Fig. 3, we use a queue of tree nodes, which expands the tree in breadth-first order. In addition, we maintain a set $V$ of non-leaf nodes, which initially only contains the root. The main difference between our method and [18] is that we adopt different criteria to decide when to add a node to the non-leaf set $V$. In [18], the set $V$ is maintained by calculating the span of vector space. However, for the language inclusion check, we cannot simply use the same criteria.

In each iteration, we remove a node $\left(\boldsymbol{v}_{1}, \boldsymbol{v}_{2}, w\right)$ from the head of queue. We then expand the tree by appending a set of its child nodes $\left(\boldsymbol{v}_{1}^{\prime}, \boldsymbol{v}_{2}^{\prime}, w^{\prime}\right)$, where $\boldsymbol{v}_{1}^{\prime}:=\boldsymbol{v}_{1} \mathbf{P}_{1}(a)$, $\boldsymbol{v}_{2}^{\prime}:=\boldsymbol{v}_{2} \mathbf{P}_{2}(a)$ and $w^{\prime}:=w a$ for all actions $a \in \alpha$. If there is a node $\left(\boldsymbol{v}_{1}^{\prime}, \boldsymbol{v}_{2}^{\prime}, w^{\prime}\right)$ such that $\operatorname{Pr}_{1}\left(w^{\prime}\right)=\boldsymbol{v}_{1}^{\prime} \boldsymbol{\kappa}_{1}>\boldsymbol{v}_{2}^{\prime} \boldsymbol{\kappa}_{2}=\operatorname{Pr}_{2}\left(w^{\prime}\right)$, then the algorithm terminates and returns $w^{\prime}$ as a counterexample for $A_{1} \sqsubseteq A_{2}$. Otherwise, we check if we can prune each child node $\left(\boldsymbol{v}_{1}^{\prime}, \boldsymbol{v}_{2}^{\prime}, w^{\prime}\right)$ (i.e. make it a leaf node) by seeing if it satisfies either of the following two criteria: (C1) $\boldsymbol{v}_{1}^{\prime} \boldsymbol{\kappa}_{1}=0$; (C2) There exist $|V|$ non-negative rational numbers $\rho^{i}$ such that, for all $\left(\boldsymbol{v}_{1}^{i}, \boldsymbol{v}_{2}^{i}, w^{i}\right) \in V, \boldsymbol{v}_{1}^{\prime} \leq \sum_{0 \leq i<|V|} \rho^{i} \boldsymbol{v}_{1}^{i}$ and $\boldsymbol{v}_{2}^{\prime} \geq \sum_{0 \leq i<|V|} \rho^{i} \boldsymbol{v}_{2}^{i}$, where $\leq$ and $\geq$ denote pointwise comparisons between vectors.

Criterion $(\mathrm{C} 1)$ is included because it is never possible to find a counterexample word with accepting probability less than $\boldsymbol{v}_{1}^{\prime} \boldsymbol{\kappa}_{1}=0$. Criterion (C2) is included because any node satisfying it would guarantee $\boldsymbol{v}_{1}^{\prime} \boldsymbol{\kappa}_{1} \leq \boldsymbol{v}_{2}^{\prime} \boldsymbol{\kappa}_{2}$; moreover, if the algorithm terminates and a node satisfies (C2), all of its descendants also satisfy (C2). We can thus make it a leaf node. In practice, (C2) can easily be checked using an SMT solver. If a node cannot be pruned, we add it to the tail of queue and to the non-leaf set $V$. The algorithm terminates if queue becomes empty, concluding that $A_{1} \sqsubseteq A_{2}$.

Correctness and termination. The correctness of the semi-algorithm in Fig. 3 is shown formally in [8]. A guarantee of termination, on the other hand, cannot be expected due to the undecidability of the underlying problem.

\section{$5 \quad L^{*}$-Style Learning for PFAs}

In this section, we propose a novel method to learn a PFA for a target weighted language generated by an unknown PFA. It works in a similar style to the well-known L* algorithm [2] for learning regular languages: it constructs an observation table (of acceptance probabilities for each word) based on two types of queries posed to a teacher. Membership queries ask the probability of accepting a particular word in the target PFA; equivalence queries ask whether a hypothesised PFA yields exactly the target language.

Fig. 4 shows the learning algorithm. It builds an observation table $(P, E, T)$, where $P$ is a finite, non-empty, prefix-closed set of words, $E$ is a finite, non-empty, suffixclosed set of words and $T:((P \cup P \cdot \alpha) \cdot E) \rightarrow[0,1]$ maps each word to its accepting probability in the target language $(\cdot$ denotes concatenation over sets). The rows of table $(P, E, T)$ are labelled by elements in the prefix set $P \cup P \cdot \alpha$ and the columns are labelled by elements in the suffix set $E$. The value $T(u \cdot e)$ of the entry at row $u$ and column $e$ is the acceptance probability of the word $u \cdot e$. We use $\operatorname{row}(u)$ to represent the $|E|$ dimensional row vector in the table labelled by the prefix $u \in(P \cup P \cdot \alpha)$.

Inspired by [4], which gives an $\mathrm{L}^{*}$-style algorithm for learning multiplicity automata, we define the notions of closed and consistent observation tables by estab- 


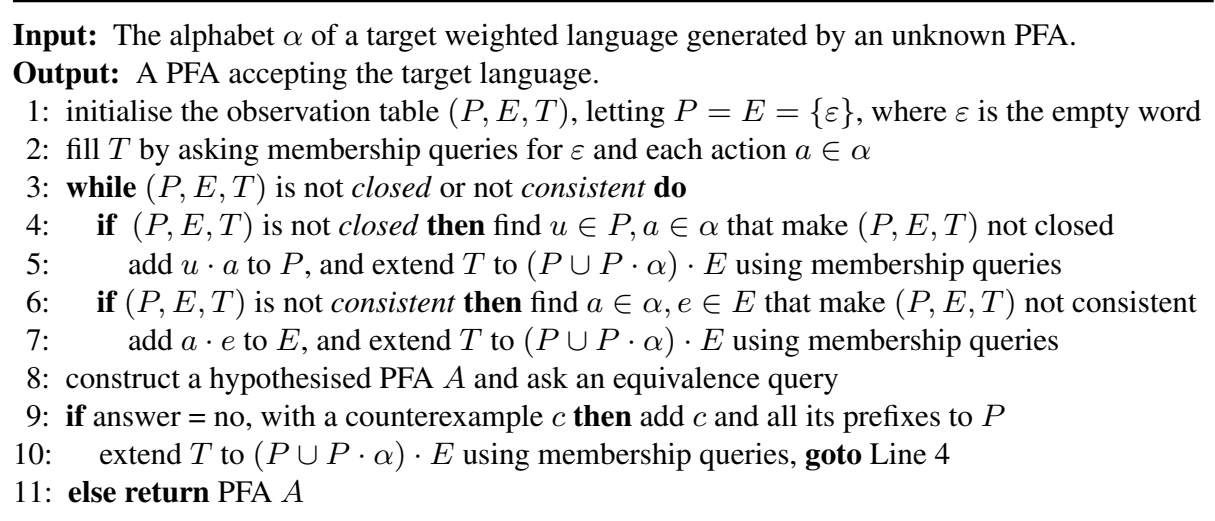

Fig. 4. $L^{*}$-style learning algorithm for PFAs

lishing linear dependencies between row vectors. Observation table $(P, E, T)$ is closed if, for all $u \in P$ and $a \in \alpha$, there exist non-negative rational coefficients $\phi_{i}$ such that $\operatorname{row}(u \cdot a)=\sum_{u_{i} \in P} \phi_{i} \operatorname{row}\left(u_{i}\right)$ and consistent if, for any rational coefficients $\psi_{i}$, $\forall e \in E . \sum_{u_{i} \in P} \psi_{i} T\left(u_{i} \cdot e\right)=0$ implies $\forall a \in \alpha, e \in E . \sum_{u_{i} \in P} \psi_{i} T\left(u_{i} \cdot a \cdot e\right)=0$. The need for coefficients to be non-negative (for closed) is a stronger condition than in [4].

As shown in Fig. 4, the observation table is filled with the results of membership queries until it is both closed and consistent. At each step, if $(P, E, T)$ is not closed (resp. consistent), then the algorithm finds $u \in P, a \in \alpha$ (resp. $a \in \alpha, e \in E$ ) that make it not closed (resp. consistent), according to the definitions above, and adds $u . a$ (resp. a.e) to the table. When $(P, E, T)$ is closed and consistent, the learning algorithm builds a hypothesis PFA $A$ (see below) and poses an equivalence query. If the teacher answers "no" (that $A$ does not yield the target language), a counterexample $c \in \alpha^{*}$ is given, for which $\operatorname{Pr}^{A}(c)$ is incorrect. The algorithm adds $c$ and all its prefixes to $P$, updates the observation table and continues to check if the table is closed and consistent. If the teacher answers "yes", the algorithm terminates and returns $A$.

Construction of a hypothesis PFA $A=(S, \bar{s}, \alpha, \mathbf{P})$, from a closed and consistent table $(P, E, T)$, proceeds as follows. First, we find a subset of $P$, denoted $\operatorname{con}(P)$, such that every element of $\{\operatorname{row}(u) \mid u \in P\}$ can be represented as a conical combination of elements in $\{\operatorname{row}(v) \mid v \in \operatorname{con}(P)\}$, i.e. there are non-negative rational coefficients $\lambda_{i}$ such that, for all $u \in P, \operatorname{row}(u)=\sum_{v_{i} \in \operatorname{con}(P)} \lambda_{i} \operatorname{row}\left(v_{i}\right)$. The set of states in the PFA is then $S=\left\{s_{0}, \ldots, s_{n-1}\right\}$, where each state $s_{i}$ corresponds to a row vector in $\{\operatorname{row}(v) \mid v \in \operatorname{con}(P)\}$ and the initial state $\bar{s}$ corresponds to $\operatorname{row}(\varepsilon)$. To obtain $\mathbf{P}(a)$ for each $a \in \alpha$, we compute, for $s_{i} \in S$, rational coefficients $\gamma_{j}$ such that $\operatorname{row}\left(s_{i} \cdot a\right)=$ $\sum_{s_{j} \in S} \gamma_{j}$ row $\left(s_{j}\right)$ and then define $\mathbf{P}(a)\left[s_{i}, s_{j}\right]:=\gamma_{j} \cdot\left(T\left(s_{j} \cdot \varepsilon\right) / T\left(s_{i} \cdot \varepsilon\right)\right)$.

Correctness and termination. When the learning algorithm terminates, it returns a correct PFA, as guaranteed by the equivalence query check. Unfortunately, we cannot prove the termination of our method. For $\mathrm{L}^{*}$, the corresponding proof uses the existence of a unique minimal DFA for a regular language. However, an analogous property does not exist for weighted languages and PFAs. According to [4], the smallest multiplicity automaton can be learnt given a weighted language. However, as shown in [6], converting a multiplicity automaton to a PFA (even for the subclass that define stochastic languages) is not always possible. 


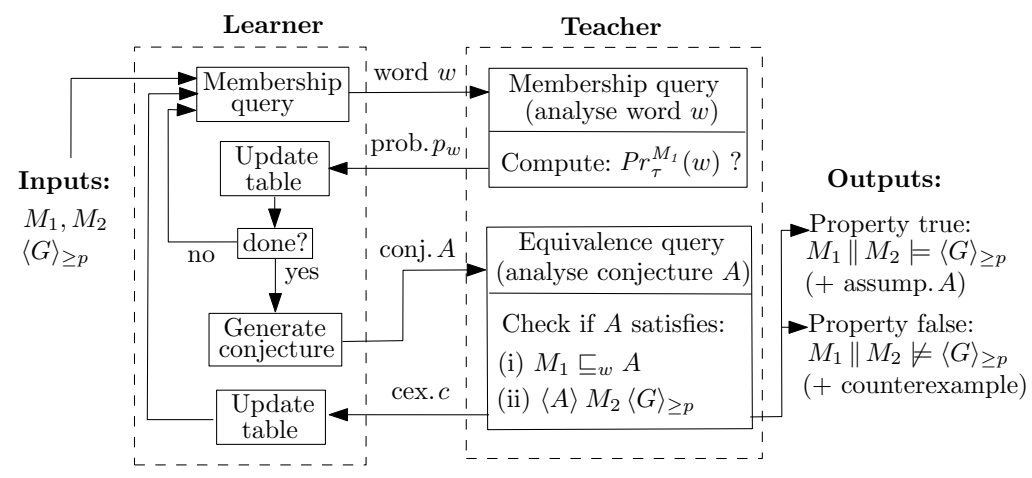

Fig. 5. L*-style PFA learning loop for probabilistic assumption generation.

\section{Learning Assumptions for Compositional Verification}

Finally, we build upon the techniques introduced in Sections 4 and 5 to produce a fully-automated implementation of the assume-guarantee framework proposed in Section 3 In particular, we use PFA learning to automatically generate assumptions to perform compositional verification. Fig. 5 summarises the overall structure of our approach, which aims to verify (or refute) $M_{1} \| M_{2} \models\langle G\rangle_{\geqslant p}$ for two PIOSs $M_{1}, M_{2}$ and a probabilistic safety property $\langle G\rangle_{\geqslant p}$. This is done using proof rule (ASYM-PIOS) from Section 3 , with the required assumption PFA $A$ about component $M_{1}$ being generated through learning. The left-hand side of the figure shows the learning algorithm of Section 5. which drives the whole process; the right-hand side shows the teacher.

The teacher answers membership queries (about word $w$ ) by computing the probability $\operatorname{Pr}_{\tau}^{M_{1}}(w)$ of word $w$ in $M_{1}$. It answers equivalence queries (about conjectured PFA $A$ ) by checking if $A$ satisfies both premises of rule (ASYM-PIOS): (i) $M_{1} \sqsubseteq_{w} A$, and (ii) $\langle A\rangle M_{2}\langle G\rangle_{\geqslant p}$. The first is done using Proposition 1 and the algorithm in Section 4. The second is done using Proposition 2, which reduces to probabilistic model checking of the DTMC $\operatorname{pios}(A) \| M_{2}$.

If both premises are true, we can conclude that $M_{1} \| M_{2} \models\langle G\rangle_{\geqslant p}$ holds. Otherwise, the teacher needs to provide a counterexample $c$ for the learning algorithm to update the observation table and proceed. If premise (i) failed, then $c$ is taken as the word showing the violation of (weak) language inclusion. If premise (ii) failed, we try to extract $c$ from the results of model checking. We extract a probabilistic counterexample [11] $C$ : a set of paths showing $\operatorname{pios}(A)|| M_{2} \not \models\langle G\rangle_{\geqslant p}$. Following the same approach as [9], we transform $C$ into a (small) fragment of $M_{1}$ (denoted $M_{1}^{C}$ ) and check whether $M_{1}^{C}|| M_{2} \not \mid\langle G\rangle_{\geqslant p}$. If so, we stop the learning loop, concluding that $M_{1}|| M_{2} \not \models\langle G\rangle_{\geqslant p}$. If, on the other hand, $C$ is a spurious counterexample, we can always extract, from $C$ a counterexample (word) $c$ such that the learning algorithm can update its observation table. Full details can be found in the extended version of this paper [8].

From the arguments above, we can show that, when the learning loop terminates, it always yields a correct result. It should be pointed out, though, that since the loop is driven by the learning algorithm of Section 5 , whose termination we cannot prove, we are also unable to guarantee that the loop finishes. Furthermore, weak language inclusion checks use the semi-algorithm of Section 4 , which is not guaranteed to terminate. 


\section{Implementation and Results}

We have implemented the PFA language inclusion check from Section 4 the PFA learning algorithm from Section 5 and the assumption-generation loop described in Section 6. Based on these, we have built a prototype tool that performs fully-automated assume-guarantee verification, as described in Section 3. Due to space limitations, we refer the reader to [8] for further details of this implementation, as well as experimental results from its application to several benchmark case studies.

Acknowledgments. The authors are supported by ERC Advanced Grant VERIWARE, EU FP7 project CONNECT and EPSRC grant EP/F001096/1. We also thank Taolue Chen, Stefan Kiefer, Björn Wachter and James Worrell for insightful discussions.

\section{References}

1. de Alfaro, L., Henzinger, T., Jhala, R.: Compositional methods for probabilistic systems. In: Proc. CONCUR'01. LNCS, vol. 2154. Springer (2001)

2. Angluin, D.: Learning regular sets from queries and counterexamples. Information and Computation 75(2), 87-106 (1987)

3. Baier, C., Katoen, J.P.: Principles of Model Checking. MIT Press (2008)

4. Bergadano, F., Varricchio, S.: Learning behaviors of automata from multiplicity and equivalence queries. SIAM J. Comput. 25(6), 1268-1280 (1996)

5. Blondel, V., Canterini, V.: Undecidable problems for probabilistic automata of fixed dimension. Theory of Computing Systems 36, 231-245 (2001)

6. Denis, F., Esposito, Y.: Learning classes of probabilistic automata. In: COLT (2004)

7. Doyen, L., Henzinger, T.A., Raskin, J.F.: Equivalence of labeled Markov chains. Int. J. Found. Comput. Sci. 19(3), 549-563 (2008)

8. Feng, L., Han, T., Kwiatkowska, M., Parker, D.: Learning-based compositional verification for synchronous probabilistic systems. Tech. Rep. RR-11-05, Department of Computer Science, University of Oxford (2011)

9. Feng, L., Kwiatkowska, M., Parker, D.: Compositional verification of probabilistic systems using learning. In: Proc. QEST'10. pp. 133-142. IEEE CS Press (2010)

10. Forejt, V., Kwiatkowska, M., Norman, G., Parker, D., Qu, H.: Quantitative multi-objective verification for probabilistic systems. In: Proc. TACAS'11 (2011)

11. Han, T., Katoen, J.P., Damman, B.: Counterexample generation in probabilistic model checking. IEEE Trans. Software Eng. 35(2), 241-257 (2009)

12. de la Higuera, C., Oncina, J.: Learning stochastic finite automata. In: ICGI (2004)

13. Kwiatkowska, M., Norman, G., Parker, D., Qu, H.: Assume-guarantee verification for probabilistic systems. In: Proc. TACAS'10 (2010)

14. Pasareanu, C., Giannakopoulou, D., Bobaru, M., Cobleigh, J., Barringer, H.: Learning to divide and conquer: Applying the $\mathrm{L}^{*}$ algorithm to automate assume-guarantee reasoning. Formal Methods in System Design 32(3), 175-205 (2008)

15. Rabin, M.: Probabilistic automata. Information and Control 6, 230-245 (1963)

16. Segala, R.: Modelling and Verification of Randomized Distributed Real Time Systems. Ph.D. thesis, Massachusetts Institute of Technology (1995)

17. Tzeng, W.G.: Learning probabilistic automata and Markov chains via queries. Mach. Learn. 8, 151-166 (1992)

18. Tzeng, W.G.: A polynomial-time algorithm for the equivalence of probabilistic automata. SIAM J. Comput. 21(2), 216-227 (1992) 